\title{
Der Frühling, er wirkt und lebt
}

\author{
Spring is at Work and Alive
}

Autor

Institut

\section{Bayerl}

Klinik für Dermatologie und Allergologie, HSK, Wilhelm-Fresenius-Klinik GmbH, Städtisches Klinikum Wiesbaden, Lehrkrankenhaus der Universität Mainz

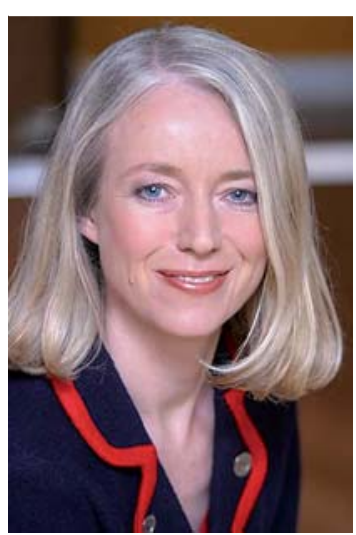

Prof. Dr. Christiane Bayerl
Bibliografie

Dol http://dx.doi.org/ 10.1055/s-0029-1243976

Akt Dermatol 2010; 36:

65 @) Georg Thieme

Verlag KG Stuttgart · New York ISSN 0340-2541

\section{Korrespondenzadresse}

Prof. Dr. med. Christiane Bayerl

Klinik für Dermatologie und

Allergologie, HSK,

Wilhelm-Fresenius-Klinik GmbH

Städtisches Klinikum Wiesbaden

Lehrkrankenhaus der

Universität Mainz

Aukammallee 39

65191 Wiesbaden

christiane.bayer!@

hsk-wiesbaden.de
Die gewogenen Leser der Aktuellen Dermatologie haben die „Blüten“ der letzten zwei Jahre freudig begrüßt, wie den Herausgebern rückgemeldet wurde. Die Reihe der CME-Artikel unter engagierter Leitung von Herrn Prof. Zillikens aus Würzburg und die Reihe Histo-Quiz unter bewährter Leitung von Frau Prof. Moll, Hamburg, sind gestartet, wurden gut angenommen und haben sich etabliert. Sie finden abwechselnd entweder einen CME-Artikel oder das Histo-Quiz in den Heften der Aktuellen Dermatologie.

In diesem Jahr geht es um eine Erweiterung und Verjüngung in der Herausgebertruppe. Es ist mir daher eine besondere Freude, Ihnen unseren Neuzugang, Frau Priv. Doz. Dr. med. habil. Gudrun Kirsten Jung vorzustellen. Frau Jung ist geboren in Mühlhausen, Thüringen, und hat ihre Weiterbildung zur Fachärztin für Immunologie am Institut für Klinische Immunologie der FriedrichSchiller-Universität Jena abgeschlossen. Ihre Dissertation erhielt das Kennzeichen mit „Auszeichnung“ und befasste sich mit allergologisch-immunochemischer Untersuchung verschiedener Pollenarten. Sie leitete das Forschungsteam zur „Herstellung und Charakterisierung monoklonaler Antikörper gegen Bienengiftbestandteile“. Ab dann kam sie in den Sog der Dermatologie. Ihre Weiterbildung zur Fachärztin für Haut- und Geschlechtskrankheiten durchlief sie an der medizinische Akademie Erfurt bzw. der Medizinischen Hochschule Erfurt. Sie leitete das allergologischimmunologische Labor und das mykologische Labor und wurde Oberärztin der Klinik und Poliklinik für Haut- und Geschlechtskrankheiten Erfurt. Die Habilitation für das Fachgebiet DermatologieVenerologie erhielt sie für das Fachgebiet Derma-

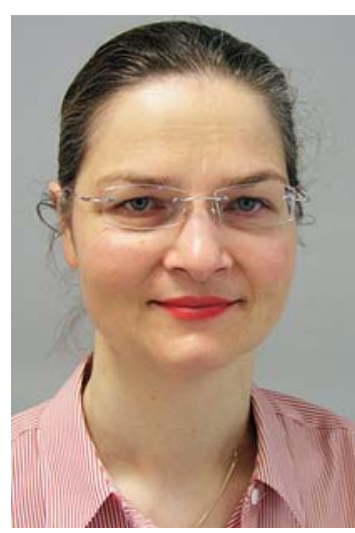

Priv. Doz. Dr. med. habil. Gudrun Kirsten Jung

tologie-Venerologie an der Medizinischen Fakultät der Friedrich-Schiller-Universität Jena. Zudem führt sie die Zusatzbezeichnung Umweltmedizin. Seit dem 1.1.2001 ist sie in Erfurt als Fachärztin für Haut- und Geschlechtskrankheiten und als Fachärztin für Immunologie niedergelassen, aktuell als „Seniorchefin“ (Zitat) einer Gemeinschaftspraxis für Dermatologie, Immunologie, Allergologie, Umweltmedizin, dermatologische Onkologie und ambulantes Operieren mit $3 \mathrm{Zu}$ lassungen und einem Weiterbildungsassistenten. Dieses Konstrukt lässt ihr etwas Zeit für Vorlesungen, Berufspolitik und Engagement für die Aktuelle Dermatologie. 2008 war Frau PD Jung Kongresspräsidentin des 3. gemeinsamen AllergieKongresses in Erfurt. Sie ist Sonderreferentin für Allergologie des Berufsverbandes der Deutschen Dermatologen e.V. und Vizepräsidentin des Ärzteverbandes Deutscher Allergologen (ÄDA).

Und damit enden meine guten Neuigkeiten nicht. Im nächsten Editorial lesen Sie die Fortsetzung. 